\title{
The Evolution of Negotiation and Impasse in Two-Party Multi-issue Bargaining
}

\author{
Fernando Lopes ${ }^{1}$, A.Q. Novais ${ }^{1}$, and Helder Coelho ${ }^{2}$ \\ ${ }^{1}$ INETI, Dep. de Modelação e Simulação, Est. Paço Lumiar, 1649-038 Lisboa, Portugal \\ \{fernando.lopes, augusto.novais\}aineti.pt \\ ${ }^{2}$ Universidade de Lisboa, Dep. de Informática, Campo Grande, 1749-016 Lisboa, Portugal \\ hcoelho@i.fc.ul.pt
}

\begin{abstract}
Automated negotiation systems are becoming increasingly important and pervasive. Most previous research on automated negotiation has focused on understanding and formalizing "successful" negotiations, i.e., negotiations that do not become contentious to the point of impasse. This paper shifts the emphasis to negotiations that are "difficult" to resolve and can hit an impasse. It analyses a situation where two agents bargain over the division of the surplus of several distinct issues to demonstrate how a procedure to avoid impasses can be utilized in a specific negotiation setting. The procedure is based on the addition of new issues to the agenda during the course of negotiation and the exploration of the differences in the valuation of these issues to capitalize on Pareto optimal agreements. This paper also lays the foundation for performing an experiment to investigate how the evolution of negotiation contributes to the avoidance of impasses, paying particular attention to the expansion of the number of issues to be deliberated and its impact on the frequency of impasses.
\end{abstract}

\section{Introduction}

Negotiation is an important and pervasive form of social interaction - it may involve two parties (bilateral negotiation) or more than two parties (multilateral negotiation), and one issue (single-issue negotiation) or many issues (multi-issue negotiation). This paper concentrates on two-party and multi-issue negotiation. We are interested in Pareto optimal outcomes (because Pareto optimality ensures that resources are not wasted), the uniqueness of these outcomes (because this allows agents to know their actual shares of the issues under dispute), and the computational complexity of different negotiation procedures and strategies (because agents should be able to negotiate in a reasonable amount of time).

Negotiation is usually understood as proceeding through three distinct phases [6]: a beginning or initiation phase, a middle or problem-solving phase, and an ending or resolution phase. The initiation phase focuses on preparation and planning for negotiation (usually referred to as pre-negotiation), and is marked by each party's efforts to posture for positions. The problem-solving phase focuses on movement toward a final agreement (usually referred to as actual negotiation), and is characterized by strategic maneuvers and jockeying for positions. The resolution phase focuses on elaborating details and implementing the final agreement.

H. Geffner et al. (Eds.): IBERAMIA 2008, LNAI 5290, pp. 213-222 2008.

(c) Springer-Verlag Berlin Heidelberg 2008 
Negotiation may end in an agreement, wherein the parties mutually agree to a proposal, or in an impasse, wherein the parties do not reach a settlement. An impasse (a stalemate or a deadlock) is a condition or state of negotiation in which there is no apparent quick or easy resolution - the parties are unable to create mutually advantageous deals that satisfy their aspirations [6]. Productive communication stops. The issues are viewed is such a way that the parties do not believe that there is any possible compatibility between them, or they cannot find a middle ground where agreement is possible. The cost of a failed or faulty negotiation can be high in many different ways (e.g., in the quantity of physical resources). Thus, effective negotiators need to understand why negotiation breaks down and be familiar with specific techniques to avoid or resolve impasses.

Artificial intelligence (AI) researchers have traditionally focused on understanding and formalizing "successful" negotiations - most researchers have assumed that negotiations result in agreement (see, e.g., [3/45]). Few researchers have attempted to formalize "difficult" negotiations, i.e., negotiations that can become contentious to the point of impasse (see, e.g., [2]7]12]). At present, despite these and other relevant pieces of work, there is still a lack of theoretical and practical understanding and important questions are still waiting to be addressed more thoroughly. We highlight the following:

- Why and how negotiations become "difficult" to resolve and reach impasse? What are the causes of impasse?

- How to manage "difficult" negotiations? Which are the actions that autonomous agents can take jointly to return negotiations to a productive phase?

- Which individual approaches to impasse avoidance or resolution are effective?

This paper addresses some of these questions in a domain-independent way.

Recently, we have proposed a model that accounts for systematic preparation and planning for negotiation, describes equilibrium strategies for the bargaining game of alternating offers, and formalizes a procedure for assisting agents to avoid impasses [9]. The procedure involves the following main actions:

- re-definition of the agenda, i.e., addition of new issues to the agenda during the course of negotiation;

- exploration of the differences in the valuation of the new issues to capitalize on Pareto optimal agreements.

Taken together, these actions add an evolutionary dimension to negotiation - some elements of the situation are not fixed, but evolve over time. Abandoning a static view simply unveils new opportunities for mutually acceptable agreements.

In this paper, we analyse a situation where two agents bargain over the division of the surplus of several distinct issues to demonstrate how the impasse avoidance procedure can be utilized in a specific negotiation setting. We also lay the foundation for performing an experiment to investigate how the evolution of negotiation contributes to the avoidance of impasses, paying particular attention to the expansion of the number of issues to be deliberated and its impact on the frequency of impasses. 


\section{Pre-negotiation}

Successful negotiators agree on one thing [613]: the keys to success in negotiation are preparation and planning (pre-negotiation). Let $A g=\left\{a g_{1}, a g_{2}\right\}$ be the set of autonomous negotiating agents. Let $I_{i}=\left\{i s_{i 1}, \ldots, i s_{i z}\right\}$ be the set of independent issues of an agent $a g_{i} \in A g$. The issues are quantitative variables, defined over continuous intervals. Negotiation usually involves a number of major or primary issues (e.g., price) and several minor or secondary issues (e.g., maintenance policies). Let $M I_{i}=\left\{i s_{i 1}, \ldots, i s_{i n}\right\}$ and $S I_{i}=\left\{i s_{i n+1}, \ldots, i s_{i z}\right\}$ be the sets of major and minor issues of $a g_{i}$, respectively. A brief description of various activities that negotiators make efforts to perform in order to carefully prepare and plan for negotiation follows (see our earlier work for an in-depth discussion [7]

Effective pre-negotiation requires that negotiators prioritize the issues, define the limits, and establish the agenda. Priorities are set by rank-ordering the issues, i.e., by defining the most important, the second most important, and so on. The priority $p r_{i l}$ of $a g_{i}$ for each issue $i s_{i l} \in I_{i}$ is a number that represents its order of preference. The weight $w_{i l}$ of $i s_{i l}$ is a number that represents its relative importance. The limit $\lim _{i l}$ is the point where $a g_{i}$ decides that it should stop to negotiate, because any settlement beyond this point is not minimally acceptable. The negotiating agenda is represented by Agenda and specifies the final set of issues to be deliberated. Its definition involves interaction with the opponent. Specifically, negotiators disclose and combine their individual sets of major issues. For the sake of simplicity, we consider that the sets $M I_{i}$ and Agenda contain the same issues.

Additionally, effective pre-negotiation requires that negotiators agree on an appropriate protocol that defines the rules governing the interaction. The protocol can be simple, allowing agents to exchange only proposals. Alternatively, the protocol can be complex, allowing agents to provide arguments to support their negotiation stance. However, most sophisticated protocols make considerable demands on any implementation, mainly because they appeal to very rich representations of the agents and their environments (see, e.g., [4]). Therefore, we consider an alternating offers protocol [10]. Two agents or players bargain over the division of the surplus of $n \geq 2$ issues (goods or pies) by alternately proposing offers at times in $\mathcal{T}=\{1,2, \ldots\}$. The negotiation procedure, labelled the "joint-offer procedure", involves bargaining over the allocation of the entire endowment stream at once. An offer is a vector $\left(x_{1}, \ldots, x_{n}\right)$ specifying a division of the $n$ goods. Once an agreement is reached, the agreed-upon allocations of the goods are implemented. This procedure permits agents to exploit the benefits of trading concessions on different issues.

The players' preferences are modelled by assuming that each player $a g_{i}$ discounts future payoffs at some given rate $\delta_{i}^{t}, 0<\delta_{i}^{t}<1,\left(\delta_{i}^{t}\right.$ is referred to as the discount factor). The cost of bargaining derives from the delay in consumption implied by a rejection of an offer. Practically speaking, the justification for this form of preferences takes into account the fact that money today can be used to make money tomorrow. Let $U_{i}$ be the payoff function of $a g_{i}$. For simplicity and tractability, we assume that $U_{i}$ is separable in all their arguments and that the per-period delay costs are the same for all issues:

$$
U_{i}\left(x_{1}, \ldots, x_{n}, t\right)=\delta_{i}^{(t-1)} \sum_{l=1}^{n} w_{i l} u_{i l}\left(x_{l}\right)
$$


where $w_{i l}$ is the weight of $i s_{i l}$ and $x_{l}$ denotes the share of $a g_{i}$ for $i s_{i l}$. The component payoff function $u_{i l}$ for $i s_{i l}$ is a continuous, strictly monotonic, and linear function. The distinguish feature of time preferences with a constant discount rate is the linearity of the function $u_{i l}$ [10]. The payoff of disagreement is normalized at 0 for both players.

Finally, effective pre-negotiation requires that negotiators be able to select an appropriate strategy. Traditionally, AI researchers have paid little attention to this pre-negotiation step. In the last several years, however, a number of researchers have developed models that include libraries of negotiation strategies (see, e.g., [3/45]). Some strategies are in equilibrium, meaning that no designer will benefit by building agents that use any other strategies when it is known that some agents are using equilibrium strategies (see, e.g., [10] for an in-depth description of the standard game-theoretic concept of equilibrium). Thus, for some situations, the agents can be designed to adopt equilibrium strategies and to negotiate according to these strategies. This paper follows this line of work.

\section{Actual Negotiation}

The negotiation process is modelled as an extensive game. For convenience, we consider the standard game-theoretic situation of two players completely informed about the various aspects of the game. The players are assumed to be rational, and each player knows that the other acts rationally. Also, we consider a particular setting in which the players have different evaluations of the issues, as described below.

Two players are jointly endowed with a single unit of each of four goods, $\left\{X_{1}, \ldots, X_{4}\right\}$, and alternate proposals until they find an agreement. Each good is modelled as an interval $[0,1]$ (or as a divisible pie of size 1). The players' preferences are as follows:

$$
\begin{aligned}
& U_{i}=\delta_{i}^{(t-1)}\left(a x_{1}+b x_{2}+x_{3}+x_{4}\right) \\
& U_{j}=\delta_{j}^{(t-1)}\left[\left(1-x_{1}\right)+\left(1-x_{2}\right)+c\left(1-x_{3}\right)+d\left(1-x_{4}\right)\right]
\end{aligned}
$$

where $x_{l}$ and $\left(1-x_{l}\right), l=1, \ldots, 4$, denote the shares of $a g_{i}$ and $a g_{j}$ for each pie, respectively. The parameters $a, b, c$, and $d$ allow the marginal utilities of the players to differ across issues and players. We consider $a>b>1$ and $d>c>1$, i.e., $a g_{i}$ places greater emphasis on goods $X_{1}$ and $X_{2}$ while $a g_{j}$ values goods $X_{3}$ and $X_{4}$ more. Also, we consider that $\delta_{i}$ and $\delta_{j}$ are close to 1 and the parameters $a, b, c$, and $d$ are close to one another. Let $p_{j \rightarrow i}^{t-1}$ and $p_{i \rightarrow j}^{t}$ denote the offers that $a g_{j}$ proposes to $a g_{i}$ in period $t-1$ and $a g_{i}$ proposes to $a g_{j}$ in period $t$, respectively. Consider the following strategies:

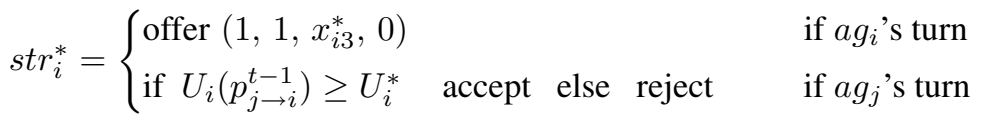

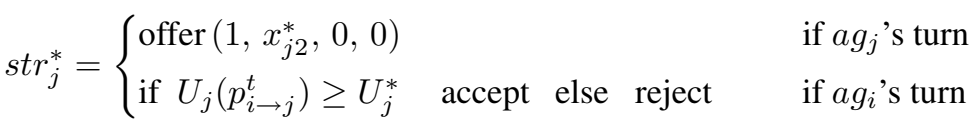

$\begin{array}{lccr}\text { where } \quad U_{i}^{*}=U_{i}\left(1, x_{j 2}^{*}, 0,0\right), \quad U_{j}^{*}=U_{j}\left(1,1, x_{i 3}^{*}, 0\right), \quad \text { and the } & \text { shares } \\ \text { are the following: } \quad x_{i 3}^{*}=\frac{\delta_{i} \delta_{j}(a+b)-\delta_{j}(a+b+b c+b d)+b c+b d}{b c-\delta_{i} \delta_{j}} & \text { and } \\ x_{j 2}^{*}=\frac{\delta_{i}\left(\delta_{i} \delta_{j}(a+b)-\delta_{j}(a+b+b c+b d)+b c+b d\right)+\left(b c-\delta_{i} \delta_{j}\right)\left(a \delta_{i}+b \delta_{i}-a\right)}{b\left(b c-\delta_{i} \delta_{j}\right)} . & \end{array}$ 
Remark 1. For the two-sided four-issue bargaining game of alternating offers with an infinite horizon, in which the players' preferences are as described above, the pair of strategies $\left(s t r_{i}^{*}, s t r_{j}^{*}\right)$ form an equilibrium. The outcome is the following:

$$
x_{1}^{*}=1, \quad x_{2}^{*}=1, \quad x_{3}^{*}=\frac{\delta_{i} \delta_{j}(a+b)-\delta_{j}(a+b+b c+b d)+b c+b d}{b c-\delta_{i} \delta_{j}}, \quad x_{4}^{*}=0
$$

Agreement is immediately reached with no delay. The outcome is Pareto optimal. Letting $\delta_{i} \rightarrow 1$ and $\delta_{j} \rightarrow 1$, the equilibrium division is $(1,1,0,0)$.

The formal proof is based on the familiar necessary conditions for equilibrium: $a g_{i}$ is indifferent between waiting one period to have its offer accepted and accepting $a g_{j}$ 's offer immediately, and $a g_{j}$ is indifferent between waiting one period to have its offer accepted and accepting $a g_{i}$ 's offer immediately. Let $\mathbf{x}_{i}^{*}=\left(x_{i 1}^{*}, \ldots, x_{i 4}^{*}\right)$ and $\mathbf{x}_{j}^{*}=\left(x_{j 1}^{*}, \ldots, x_{j 4}^{*}\right)$ be the equilibrium proposals of $a g_{i}$ and $a g_{j}$, respectively. The problem for $a g_{i}$ is to find an offer that maximizes its payoff (because it is a payoff maximizer) subject to being acceptable to its opponent, i.e.,

maximize:

$$
U_{i}\left(x_{1}, \ldots, x_{4}, t\right)=\delta_{i}^{(t-1)}\left(a x_{1}+b x_{2}+x_{3}+x_{4}\right)
$$

subject to:

$$
\begin{aligned}
& \left(1-x_{i 1}^{*}\right)+\left(1-x_{i 2}^{*}\right)+c\left(1-x_{i 3}^{*}\right)+d\left(1-x_{i 4}^{*}\right)= \\
& \delta_{j}\left[\left(1-x_{j 1}^{*}\right)+\left(1-x_{j 2}^{*}\right)+c\left(1-x_{j 3}^{*}\right)+d\left(1-x_{j 4}^{*}\right)\right] \\
& 0 \leq x_{i l}^{*} \leq 1, \quad 0 \leq x_{j l}^{*} \leq 1, \quad \text { for } l=1, \ldots, 4
\end{aligned}
$$

The problem for $a g_{j}$ is stated in a similar way and is omitted. Solving both maximization problems yields the outcome specified in the statement of the Remark. In the limit, letting $\delta_{i} \rightarrow 1$ and $\delta_{j} \rightarrow 1$, the outcome of the equilibrium is $(1,1,0,0)$. This outcome is on the Pareto frontier and corresponds to the utility pair $(a+b, c+d)$.

\section{Bargaining Impasse}

Negotiators can adopt different orientations (and strategies) to accomplish their goals. Two bargaining orientations are commonly discussed in the literature [11]: individualistic or competitive and cooperative or problem solving. Individualistic negotiators show a strong interest in achieving only their own outcomes - getting this deal, winning this negotiation - and tend to pursue competitive strategies (e.g., appearing firm and imposing time pressure). Cooperative negotiators are concerned with both their own and the other's outcomes - building, preserving, or enhancing a good relationship with the other party - and tend to pursue problem solving strategies (e.g., logrolling and compensation).

The last section has considered a typical bargaining situation of two cooperative agents and a "win-win" philosophy (formalized, at least in part, by the strategic choices of the players). The agents were assumed to be sufficiently creative to devise the Pareto frontier and able to settle for the outcome that maximizes their benefit (resources were not wasted and money was not squandered). 
This section addresses a different bargaining situation - it considers a cooperative agent, say $a g_{i}$, and a competitive agent, $a g_{j}$, who wants to "win" the negotiation. Now, $a g_{j}$ pursues a strategy compatible with its negotiating style (e.g., starting with high demands and making a few small concessions throughout negotiation [7/8]). In period 1 , $a g_{i}$ proposes the offer $\mathbf{x}_{i}^{*}$ specified in Remark 1 , and $a g_{j}$ either accepts this offer or rejects it. We restrict attention to the case in which $a g_{j}$ rejects the offer $\mathbf{x}_{i}^{*}$. The play passes to period 2 and $a g_{j}$ proposes an offer $\mathbf{y}_{j}$, which $a g_{i}$ rejects (considering that $\left.U_{i}\left(\mathbf{y}_{j}, 2\right)<U_{i}\left(\mathbf{x}_{i}^{*}, 3\right)\right)$. The agents continue to negotiate in this manner and, therefore, negotiation can become "difficult" to the point of impasse.

In this situation, $a g_{i}$ can try to draw $a g_{j}$ into a more constructive process. Specifically, $a g_{i}$ can manage the sets of major and minor issues constructively by performing the following actions:

- analysis of the set $S I_{i}$ of minor issues; selection of issues that are believed to cost less than they are worth to $a g_{j}$;

- addition of new issues to the set $M I_{i}$ of major issues (and subsequent re-definition of the agenda);

- exploration of the differences in the valuation of the new issues.

The basic idea is to allow $a g_{i}$ to prepare a new proposal that maximizes its benefit and simultaneously is more favourable to $a g_{j}$ (than any previous proposal). Taken together, the suggested actions add an evolutionary dimension to the analysis. They can enable the enlargement of the space of feasible settlements, thus facilitating movement towards an optimal agreement.

Consider a new two-sided five-issue bargaining situation obtained from the two-sided four-issue situation introduced in the previous section by changing the agenda, i.e., by adding a new good $X_{5}$. For the new bargaining game of alternating offers, time starts at period 1. However, the players' preferences take into account the costs of bargaining derived from the delays in consumption implied by the rejection of offers in the "initial" situation, i.e.,

$$
\begin{aligned}
& U_{i}=\delta_{i}^{(t-1)+\tau}\left(a x_{1}+b x_{2}+x_{3}+x_{4}\right)+\delta_{i}^{(t-1)} x_{5} \\
& U_{j}=\delta_{j}^{(t-1)+\tau}\left[\left(1-x_{1}\right)+\left(1-x_{2}\right)+c\left(1-x_{3}\right)+d\left(1-x_{4}\right)\right]+\delta_{j}^{(t-1)} e\left(1-x_{5}\right)
\end{aligned}
$$

where $\tau$ is a time period, $a>b>1$ and $d>c>e>1$. Consider the following strategies:

$$
\begin{aligned}
& s t r_{i}^{* *}= \begin{cases}\text { offer }\left(1,1,0,0, x_{i 5}^{* *}\right) & \text { if } a g_{i} \text { 's turn } \\
\text { if } U_{i}\left(p_{j \rightarrow i}^{t-1}\right) \geq U_{i}^{* *} \quad \text { accept else reject } & \text { if } a g_{j} \text { 's turn }\end{cases}
\end{aligned}
$$

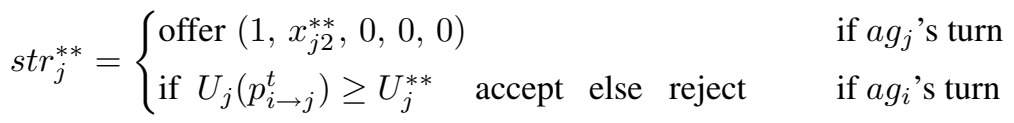

where $\quad U_{i}^{* *}=U_{i}\left(1, x_{j 2}^{* *}, 0,0,0\right), \quad U_{j}^{* *}=U_{j}\left(1,1,0,0, x_{i 5}^{* *}\right)$,

$x_{i 5}^{* *}=\frac{(a+b) \delta_{i}^{\tau} \delta_{j}^{\tau+1}-(a+b+b c+b d) \delta_{i}^{\tau-1} \delta_{j}^{\tau+1}+(b c+b d) \delta_{i}^{\tau-1} \delta_{j}^{\tau}-b e \delta_{i}^{\tau-1}\left(\delta_{j}-1\right)}{b e \delta_{i}^{\tau-1}-\delta_{j}^{\tau+1}}$, and

$x_{j 2}^{* *}=\frac{\left(a b e+b^{2} e\right) \delta_{i}^{\tau}-a b e \delta_{i}^{\tau-1}-(b+b c+b d) \delta_{j}^{\tau+1}+(b c+b d) \delta_{j}^{\tau}-b e\left(\delta_{j}-1\right)}{b\left(b e \delta_{i}^{\tau-1}-\delta_{j}^{\tau+1}\right)}$. 
Remark 2. For the new bilateral five-issue bargaining game of alternating offers with an infinite horizon, in which the players' preferences are as described above, the pair of strategies $\left(s t r_{i}^{* *}, s t r_{j}^{* *}\right)$ form an equilibrium. The outcome is Pareto optimal:

$$
\begin{aligned}
& x_{1}^{* *}=1, \quad x_{2}^{* *}=1, \quad x_{3}^{* *}=0, \quad x_{4}^{* *}=0, \\
& x_{5}^{* *}=\frac{(a+b) \delta_{i}^{\tau} \delta_{j}^{\tau+1}-(a+b+b c+b d) \delta_{i}^{\tau-1} \delta_{j}^{\tau+1}+(b c+b d) \delta_{i}^{\tau-1} \delta_{j}^{\tau}-b e \delta_{i}^{\tau-1}\left(\delta_{j}-1\right)}{b e \delta_{i}^{\tau-1}-\delta_{j}^{\tau+1}}
\end{aligned}
$$

Agreement is immediately reached with no delay. Letting $\delta_{i} \rightarrow 1$ and $\delta_{j} \rightarrow 1$, the equilibrium division is $(1,1,0,0,0)$.

The formal proof is similar to the proof of Remark 1. At this stage, it is worth noting that the offer specified in Remark 2 is more favourable to $a g_{j}$ than the offer specified in Remark 1. Thus, this new offer can be accepted, i.e., the negotiation process may end successfully and the Pareto optimal agreement may be implemented.

Example 1. Consider a sales agent and a logistics agent operating in a multi-agent supply chain system. The sales agent is responsible for acquiring orders from customers, negotiating with customers, and handling customer requests for modifying or canceling orders. The logistics agent is responsible for coordinating the plants and distribution centers of a manufacturing enterprise - it manages the movement of materials and products across the supply chain, from the suppliers of raw materials to the customers of finished goods. In particular, consider the following situation:

"David, the director of Sales, is trying to arrange for production of its two new orders, one for 10000 and the other for 5000 men's suits. Martin, the director of Logistics, is stating that the job will take four months. Together, they will gross over 25000 Euros, with a fine profit for the company. The problem is that Martin insists that the job will take four months and David's customer wants a two-month turnaround. Also, David claims that it can't afford to lose the customer".

There are four major issues of concern in negotiation, namely quantity_1 and date_1 (for the 10000 suit order), and quantity 2 and date 2 (for the 5000 suit order). The sales agent places greater emphasis on quantity_1 and date_1 (due to the inherent customer demands). On the other hand, the logistics agent values quantity_2 and date_2 more. Figure 1 shows the joint utility space for the agents (the small squares represent a few possible agreements). The Pareto frontier is represented by the dotted line OAO' and the optimal outcome referred to in Remark 1 by the point A. This outcome provides a (normalized) benefit of 0.65 to each party (letting $\delta_{i} \rightarrow 1$ and $\delta_{j} \rightarrow 1$ ).

Additionally, there is one minor issue for the sales agent: meeting_attendance, i.e., the right to attend a number of Sales Division meetings that are of interest to the Logistics Division. The inclusion of this issue unveils new opportunities for mutually acceptable agreements, thus changing the location of the Pareto frontier. Figure 1 shows the new location of the frontier (line OBO'). The optimal outcome referred to in Remark 2 is represented by the point $\mathrm{B}$ and provides a (normalized) benefit of 0.725 to Martin and 0.55 to David (again, letting $\delta_{i} \rightarrow 1$ and $\delta_{j} \rightarrow 1$ ). Thus, Martin may re-analyze the negotiation situation, adopt a different negotiating style, and respond favourably to this new solution. 


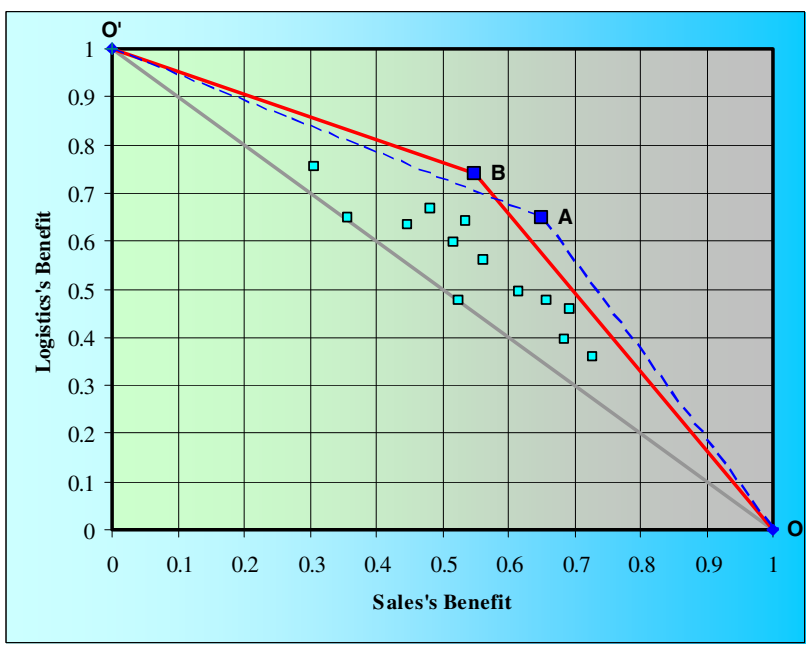

Fig. 1. Joint utility space for the sales and logistics agents

\section{Experimental Analysis: Preliminary Report}

The analytic results just given ensure the benefits of adding new issues to the agenda for the negotiation setting discussed here. However, the theory does not articulate how fast the convergence of negotiation is. Specifically, negotiation may become contentious to the point of impasse, even if the agents are sufficiently creative to re-define the agenda and exploit the differences in the valuation of the new issues to capitalize on Pareto optimal agreements. We intend to address this issue by empirically investigating how the evolution of negotiation contributes to the avoidance of impasses, paying particular attention to the expansion of the number of issues to be deliberated and its impact on the frequency of impasses. Accordingly, we describe below the experimental method, discuss the independent and dependent variables, and introduce the preliminary hypotheses. In so doing, we lay the foundation for the experimental work.

The experimental method is controlled experimentation [1]. The experiment involves three types of agents: (i) competitive agents, (ii) creative agents, and (iii) impasse avoidance agents. Competitive agents are individualistically oriented and pursue a strategy str. This strategy models an optimistic opening attitude and successive small concessions [7/8]. Creative agents are cooperatively oriented and pursue a strategy $s t{ }^{*}$ (see Remark 1). Impasse avoidance agents are also cooperatively oriented and pursue a strategy $s t r^{*}$ at the beginning of negotiation and a strategy $s t r^{* *}$ after the rejection of the first offer (see Remark 2).

Research on human negotiation has shown that motivational orientation (and strategy) affects both process and outcome in bilateral interaction (see, e.g., [1113]). A cooperative orientation has increased the joint benefit of negotiators. Also, when aspirations were high and inflexible, negotiations were more likely to be broken off under an individualistic as opposed to a cooperative orientation. Accordingly, the hypotheses are as follows (a dyad is composed by a sales agent and a logistics agent): 
Hypothesis 1. Dyads whose sales agent is cooperatively oriented (cooperatively oriented dyads) will reach higher quality outcomes (higher joint benefits) than dyads whose sales agent is individualistic oriented (individualistic oriented dyads).

Hypothesis 2. The impasse rate will be lower in cooperatively oriented dyads than in individualistic oriented dyads. The impasse rate will be lowest in cooperatively oriented dyads composed by an impasse avoidance sales agent.

Hypothesis 3. The cost of bargaining (the time spent in negotiation) will be lower in cooperatively oriented dyads composed by an impasse avoidance sales agents than in cooperatively oriented dyads composed by a creative sales agent.

The independent variable is the bargaining orientation of the sales agent (manipulated by assigning a specific strategy to this agent). This variable has three levels, namely the three strategies mentioned earlier. The dependent variables are the impasse rate, the time spent in negotiation, the Pareto efficiency, and the joint benefit provided by the final agreement. The first dependent variable is the impasse rate, i.e., the frequency of impasses. The second variable is the time spent in negotiation (measured in terms of the total number of offers exchanged by the agents). The next variable is the Pareto efficiency, i.e., the extent to which an agreement approaches the frontier (a fully Pareto efficient agreement is Pareto optimal). The last dependent variable is the joint benefit provided by the final agreement, i.e., the sum of the two agents' benefits in the final agreement.

The experiment involves three groups of trials. Each group corresponds to a level of the independent variable. A trial is a single run of the experimental system and involves a bargaining session. Trials of the same group will, in general, differ from one another. The detailed experimental procedure is a follows:

- for each group of trials, the experimenter manipulates the independent variable, i.e., assigns a strategy to the sales agent;

- for each trial in each group, the experimenter randomly determines the agent that starts the bidding process and the orientation of the logistics agent, i.e., its strategy (from a library containing competitive and problem solving strategies [7] [8]);

- for all trials of each group, the experimenter measures the dependent variables and computes averages on the measures taken.

\section{Related Work}

Traditionally, AI researchers have focused on modelling "successful" negotiations, i.e., negotiations that result in agreement (see, e.g., [3]4[5]). There are, however, some researchers that have studied various techniques that autonomous agents can use to resolve impasses on their own (see, e.g., [2]7|12]). Faratin [2], for example, presented a model that incorporates a mechanism to assist agents in dynamically including or retracting issues from the set of negotiation issues. The author acknowledged the usefulness of the mechanism to escape negotiation deadlocks. However, he pointed out that the mechanism is complex and deferred to future work both its specification and its empirical analysis. Lopes et al. [7] developed a model that formalizes a structure for the problem under negotiation and supports the dynamic change of that structure to achieve movement towards agreement (problem restructuring). The authors pointed out that 
problem restructuring facilitates the resolution of impasses. However, they observed that problem restructuring is a highly creative and challenging task and postponed its formal treatment to future work. Sycara [12] developed the Persuader system for resolving conflicts in the domain of labor relations. Problem restructuring may take place during the modification of a proposal.

At present, despite these and other relevant pieces of work, the study of negotiations that are "difficult" to resolve, the formalization of approaches to impasse avoidance, and the empirical evaluation of agents capable of managing "difficult" negotiations are still in its infancy. This paper has addressed these issues in a specific negotiation setting.

\section{Conclusion}

This paper has shifted the emphasis to negotiations that are "difficult" to resolve and can hit an impasse. It has analysed a particular situation where two agents bargain over the division of the surplus of several distinct issues and has demonstrated the benefits of an impasse avoidance procedure involving the re-definition of the agenda during the course of negotiation. It has also laid the foundation for performing an experiment to investigate how the evolution of negotiation contributes to the avoidance of impasses.

Autonomous agents able to negotiate and avoid impasses under complete information are currently being developed using the Jade framework. Our aim for the future is to perform a set of experiments to validate the key components of the agents. In addition, we intend to develop more sophisticated agents that are able to negotiate and avoid impasses under incomplete information.

\section{References}

1. Cohen, P.: Empirical Methods for Artificial Intelligence. MIT Press, Cambridge (2001)

2. Faratin, P.: Automated Service Negotiation Between Autonomous Computational Agents. Ph.D. Thesis, Queen Mary \& Westfield College, UK (2000)

3. Ito, T., Hattori, H., Zhang, M., Matsuo, T.: Rational, Robust, and Secure Negotiations in Multi-Agent Systems. Springer, Heidelberg (2008)

4. Jennings, N., Faratin, P., Lomuscio, A., Parsons, S., Wooldridge, M., Sierra, C.: Automated Negotiation: Prospects, Methods and Challenges. Group Dec. and Neg. 10, 199-215 (2001)

5. Kraus, S.: Strategic Negotiation in Multi-Agent Environments. MIT Press, Cambridge (2001)

6. Lewicki, R., Barry, B., Saunders, D., Minton, J.: Negotiation. McGraw Hill, New York (2003)

7. Lopes, F., Mamede, N., Novais, A.Q., Coelho, H.: A Negotiation Model for Autonomous Computational Agents: Formal Description and Empirical Evaluation. Journal of Intelligent \& Fuzzy Systems 12, 195-212 (2002)

8. Lopes, F., Mamede, N., Novais, A.Q., Coelho, H.: Negotiation Strategies for Autonomous Computational Agents. In: ECAI 2004, pp. 38-42. IOS Press, Amsterdam (2004)

9. Lopes, F., Fatima, S., Wooldridge, M.: Pre-Negotiation and Impasse in Automated Negotiation. Group Dec. and Neg. (submitted, 2007)

10. Osborne, M., Rubinstein, A.: Bargaining and Markets. Academic Press, London (1990)

11. Pruitt, D., Kim, S.: Social Conflict: Escalation, Stalemate, and Settlement. McGraw Hill, New York (2004)

12. Sycara, K.: Problem Restructuring in Negotiation. Management Sci. 37, 1248-1268 (1991)

13. Thompson, L.: The Mind and Heart of Negotiator. Prentice-Hall, Englewood Cliffs (2005) 